\title{
Review Article \\ Recent Advances of Hemorrhage Management in Severe Trauma
}

\author{
Mohamed El Sayad ${ }^{1}$ and Hussein Noureddine ${ }^{2}$ \\ ${ }^{1}$ Trauma and Orthopaedics, Wishaw General Hospital, Wishaw, UK \\ ${ }^{2}$ Trauma and Orthopaedics, Ninewells Hospital, Dundee, UK \\ Correspondence should be addressed to Mohamed El Sayad; msayad1@hotmail.com
}

Received 3 May 2013; Revised 14 September 2013; Accepted 31 October 2013; Published 30 January 2014

Academic Editor: Chak W. Kam

Copyright (C) 2014 M. El Sayad and H. Noureddine. This is an open access article distributed under the Creative Commons Attribution License, which permits unrestricted use, distribution, and reproduction in any medium, provided the original work is properly cited.

\begin{abstract}
Trauma is one of the most common causes of mortality worldwide with a substantial percentage of deaths resulting secondary to haemorrhages, which are preventable and treatable when adequately managed. This paper offers a review of the current literature on how to successfully resuscitate patients with major haemorrhage.
\end{abstract}

\section{Introduction}

Trauma is the third most common cause of mortality worldwide and is the leading cause of death in the age group ranging between 1 and 44 years [1]. Among those trauma patients, major haemorrhage is responsible for 30 to $40 \%$ of mortality, with up to half of them dying prior to their arrival to hospital [1]. Whilst injuries to the central nervous system are the leading cause of mortality, haemorrhagic shock ranks second despite the fact that it could be preventable and reversible. Within hospital settings, it accounts for more than $80 \%$ of the mortality in operating theatres and $50 \%$ of the overall mortality in the first 24 hours of admission [1]. Major haemorrhage is defined as the loss of $100 \%$ of total blood volume within 24 hours, loss of $50 \%$ within four hours, or the loss of $150 \mathrm{~mL}$ per minute. Given the above, the importance of successful resuscitation of major haemorrhage becomes paramount [2].

\section{Early Identification}

Early identification of patients with major haemorrhage is a necessity and the diagnosis should be established promptly. Suffering from hypovolemia due to haemorrhage presents with a variety of clinical features. The American College of Surgeons through their ATLS protocols have traditionally provided a classification for haemorrhagic shock that is divided according to the amount of blood loss; see Table 1 [3]. However, there are limitations to this classification. For example, the clinical signs might be masked in young fit individuals with larger physiological reserves, as well as elderly patients on medications such as beta-blockers that manipulate normal physiological responses.

Introduction of new methods of shock assessment has shown to be more sensitive compared to conventional monitoring of vital signs. The shock index is the ratio of heart rate to systolic blood pressure. Birkhahn et al. proved that the shock index was a more useful tool in diagnosing early haemorrhage. They performed an observational study that included 46 healthy blood-donating individuals; each patient had $450 \mathrm{mLs}$ of blood taken over 20 minutes. Their vital signs were measured before donation, immediately after donation while lying down, and after one and five minutes of standing. The results showed a significant increase in the mean shock index, whilst the vital signs remained within the normal limits despite revealing some extent of variation from predonation readings [4].

Cannon et al. performed a retrospective review of 2445 trauma patients to examine the correlation between shock index and mortality rates. Results showed that patients with a shock index ratio of more than 0.9 had a higher mortality rate of $15.9 \%$ compared to a rate of $6.9 \%$ in those with a normal shock index. Furthermore, patients whose shock indices measured in the emergency department were higher than those measured in the field had a mortality rate of $9.3 \%$ compared to only $5.7 \%$ in those who had unchanged shock indices [5]. 
TABLE 1: Classification of haemorrhagic shock (ATLS manual American College of Surgeons).

\begin{tabular}{|c|c|c|c|c|}
\hline \multicolumn{5}{|c|}{ Class of haemorrhagic shock } \\
\hline & $\mathrm{I}$ & II & III & IV \\
\hline Blood loss (mL) & Up to 750 & $750-1500$ & $1500-2000$ & $>2000$ \\
\hline Blood loss (\% blood volume) & Up to 15 & $15-30$ & $30-40$ & $>40$ \\
\hline Pulse rate (per minute) & $<100$ & $100-120$ & $120-140$ & $>140$ \\
\hline Blood pressure & Normal & Normal & Decreased & Decreased \\
\hline Pulse pressure $(\mathrm{mm} \mathrm{Hg})$ & Normal or increased & Decreased & Decreased & Decreased \\
\hline Respiratory rate (per minute) & $14-20$ & $20-30$ & $30-40$ & $>35$ \\
\hline Urine output (mL/hour) & $>30$ & $20-30$ & $5-15$ & Negligible \\
\hline Central nervous system/mental status & Slightly anxious & Mildly anxious & Anxious/confused & Confused/lethargic \\
\hline
\end{tabular}

ROPE is another method of assessment that has also been described; it involves the division of pulse rate by pulse pressure (pulse rate/systolic blood pressure-diastolic blood pressure). Ardagh et al. assessed ROPE as a mean to predict the risk of decompensation in patients with compensated haemorrhagic shock, by undergoing a retrospective study on a cohort of 184 trauma victims. Their results showed that a ROPE value of less than 3.0 was predictive of patients remaining stable while a ROPE value of more than 3.0 was predictive of patients that may develop decompensated haemorrhagic shock [6].

Both methods were assessed by Campbell et al. [7] who carried out a prospective observational study of one hundred and sixteen healthy individuals that donated one unit of blood ( $420 \mathrm{mLs})$; both their heart rate and blood pressure were measured immediately before and after donating the blood. Their shock index and ROPE measurements were calculated and the mean of both measurements increased significantly by $12.2 \%$. This study showed that the rope and shock indices correlated with blood loss better than conventional observation monitoring and that they are suitable methods to detect early blood loss.

Subsequent to diagnosing hypovolemia due to haemorrhage, it becomes imperative to identify the source of bleeding. This can be achieved by clinical examination (reduced breath sounds, abdominal or pelvic tenderness, deformed limbs, etc.), bedside investigations in the emergency department (trauma series X-rays, fast scan), or radiological investigation most notably whole body CT scan which is very helpful in rapid diagnosis after patient stabilization [8].

\section{Damage Control Resuscitation (Prevention and Treatment of the Lethal Triad)}

Our approach to major haemorrhage mainly stems from experiences accumulated during periods of war. The conflicts in both Afghanistan and Iraq have inspired us to implement changes to current practice. The military has developed a trauma resuscitation protocol of their own similar to the ATLS called Battlefield Advanced Trauma Life Support (BATLS). This is applied to the management of military trauma victims where the starting point is stopping catastrophic haemorrhage, ensued by the conventional ABCDE ATLS algorithm [9].
For appropriate resuscitation to be established, it is important to understand the severe pathophysiological derangements of the exsanguinating patients, in terms of hypothermia, coagulopathy, and acidosis also known as the lethal triad. Bleeding patients with these findings have up to $90 \%$ mortality rate requiring massive transfusion [10]. We will discuss each of the elements of the lethal triad in turn.

3.1. Hypothermia. Hypothermia secondary to trauma is a common occurrence; it is due to the inability of the body to generate heat, because of altered central thermoregulation, blocked shivering response, and reduction of metabolic activity at the cellular level. Resuscitation is also a common reason for hypothermia. Cold intravenous fluid administration and patient exposure during initial evaluation as well as peritoneal exposure during laparotomies in the operating theatres are all major contributors. Hypothermia is clinically significant when the core temperature is less than 36 degrees for more than 4 hours.

Hypothermia exacerbates coagulopathy by affecting platelet function; the imbalance of thromboxane and prostacyclin reduces the response of platelet activation. Hypothermia also reduces the enzyme activation pathway of the coagulation cascade. At $35^{\circ} \mathrm{C}$, factors eleven and twelve would only function at $65 \%$ of normal [11]. Trauma victims with a core temperature below $35^{\circ} \mathrm{C}$ have a poor prognosis, and those with a core temperature of $32^{\circ} \mathrm{C}$ have a $100 \%$ mortality rate [11]. Recent data from the 31st Combat Support Hospital in Iraq showed that $18 \%$ of 2848 trauma patients were hypothermic and that hypothermia is significantly correlated with admission GCS, tachycardia, hypotension, low hematocrit, and acidosis thus making it an independent contributor to overall mortality [12].

Treatment of the hypothermic patient could be carried out passively or actively. Passive warming entails preventing further heat loss, by means such as covering the patients and warming the operating or resuscitation room. Active warming involves covering the trauma victims with warming blankets and administering warmed intravenous fluid or blood and warm body cavity lavage, aiming to keep core temperature at 36 degrees.

3.2. Acidosis. Metabolic acidosis is due to the prolonged inadequate tissue perfusion. This leads to cellular metabolism 
converting from aerobic to anaerobic and the increased production of lactate causing a reduction in $\mathrm{pH}$. This reduction in $\mathrm{pH}$ has a negative effect on cardiac contractility.

The effect of the acidosis on the coagulation cascade was shown by Meng et al. They measured the activity of recombinant factor VIIa on phospholipids and platelets, and the results showed that it was reduced by $90 \%$ when the $\mathrm{pH}$ was reduced from 7.4 to 7 [13]. Martini et al. showed the effect of acidosis on coagulation by infusing 12 pigs with $0.2 \mathrm{~mol} / \mathrm{L}$ of hydrochloric acid until their $\mathrm{pH}$ was 7.1. Ringer lactate was continually transfused to maintain that $\mathrm{pH}$. Blood samples were taken at baseline and at 15 minutes after acidosis induction. Coagulation function was assessed by measuring the prothrombin time (PT), partial thromboplastin time (PTT), and thrombin generation. They concluded that acidosis reduced fibrinogen concentration to $66 \% \pm 2 \%$, decreased platelet counts to $49 \% \pm 4 \%$, and decreased thrombin generation to $60 \% \pm 4 \%$. Acidosis also prolonged PT and PTT by about 20\% [14].

A sensitive means of assessing tissue oxygenation is by measuring the base deficit, which is a measure of the number of millimoles of base required to correct the $\mathrm{pH}$ of one liter of whole blood to 7.4 and its normal value is -3 to +3 . Davis et al. demonstrated that measurement of the base deficit was more accurate in deciding the clearance of the acidosis after trauma shock than $\mathrm{PH}$ measurement. They performed a retrospective analysis of 674 trauma victims that had both their $\mathrm{pH}$ and base deficits measured during their admission to hospital. The base deficit was significantly different between the survivors and the nonsurvivors at all of the time intervals at which it was measured. However, the same differences were not noted when the $\mathrm{pH}$ was assessed making it a less reliable predictor of outcome [15].

Treatment of acidosis mainly constitutes restoration of the circulation to maintain tissue perfusion. Lier et al. provided a comprehensive review of the effect of acidosis on coagulation and recommended neutralization of the acidosis to improve the coagulopathy especially when the trauma victim is receiving a massive transfusion. The recommendation was that buffering could be established by using either sodium bicarbonate or trishydroxymethylaminomethane (THAM), with the latter recording superior results. This is due to its effect on coagulation, as it did not inhibit thrombin formation when compared to sodium bicarbonate, but they both had a negative effect on the conversion of fibrinogen to fibrin [16].

3.3. Coagulopathy. Hess et al. reviewed the reasons for the development of acute coagulopathy following trauma. They identified 6 initiators: (1) tissue damage which leads to the exposure of endothelium and initiates the coagulation cascade and fibrinolysis; (2) shock, which is dose dependent to the degree of coagulopathy; this mechanism is not clearly understood; (3) haemodilution from the shift of cellular and interstitial fluid that is deficient in clotting factors into the plasma, and the administration of intravenous fluid, which also interrupts clot formation and the transfusion of red blood cells, (4) inflammation which interferes in coagulation as it causes monocytes to adhere to platelets, activation of the thrombomodulin-protein $\mathrm{C}$ pathway, and the binding of C4b to protein S [17], finally the last 2 initiators which are (5) hypothermia and (6) acidosis as discussed previously.

Acute coagulopathies are found in one in four trauma patients as shown by Brohi et al. They underwent a retrospective review of 1088 patients that were admitted to their trauma centre between the years of 1993 and 1998. These patients had their coagulation profile measured on admission, including PT, APTT, and thrombin time. The results showed that $24.4 \%$ of patients had a coagulopathy and that these patients had a mortality of $46 \%$. This was significantly different to the mortality of $10.9 \%$ for those with normal clotting [18].

Currently, there are no accurate methods to assess acute trauma coagulopathy. The traditional investigations of PT, PTT, fibrinogen, and platelet count are of limited value during massive hemorrhage as they do not provide enough information about clot formation. Additionally, the platelet count is usually normal during the initial stage of haemorrhage. The current treatment of coagulopathy is by the early empirical transfusion of blood and clotting factors [19].

3.4. Massive Transfusion and Fluid Resuscitation. Massive transfusion is defined as transfusion of more than ten units of packed red cells over 24 hours or four units within one hour. Administering blood should be initiated at the scene of the accident, thus avoiding the rapid administration of iv fluids (filler fluid) that has been traditionally promoted by the ATLS guidelines. This involves giving two liters of crystalloids and continuing with packed red blood cells (PRBCs) and freshfrozen plasma (FFP) if there was transient or no response, with the aim of achieving normotension.

Duke et al. provided data from a retrospective analysis of 307 patients that were admitted to a level one-trauma centre between January 2007 and May 2011. The inclusion criteria were patients with penetrating torso injuries and a systolic blood pressure less than $90 \mathrm{mmHg}$ who were managed by damage control resuscitation and surgery. 175 patients received standard fluid resuscitation (SFR) which was $150 \mathrm{mLs}$ of crystalloid or more; 132 patients received restricted fluid resuscitation (RFR) which was less than $150 \mathrm{mLs}$ of crystalloid before damage control surgery. The results showed that the first group (SFR) received more preoperative fluid then the (RFR) group $(2,275 \mathrm{mLs}$ versus $129 \mathrm{mLs})$ and that they had a higher intraoperative mortality rate (32\% versus $9 \%$ ) and overall mortality rate (37\% versus $21 \%)$. The higher mortality rate was attributed to the effect of large volume of fluids in diluting clotting factors and reducing blood viscosity and the increase of blood pressure. RFR was beneficial to these patients as it provided them with permissive hypotension (systolic blood pressure of 90) until damage control surgery was achieved [20].

Serious attempts at challenging the clinical validity of the widely accepted concept of conventional fluid resuscitation first surfaced in the mid-1990s. In 1996, Dries published a mini review of summaries in his paper "hypotensive resuscitation" suggesting that "Limited attempts to restore blood pressure improve cardiac output, tissue perfusion, and survival while attempts to restore normal tension with crystalloid result in increased hemorrhage volume and higher mortality" 
[21]. However, some of the earlier randomized controlled trials did not concur with those findings. For instance, Dutton et al. [22] underwent a study where a cohort of 110 patients presenting in hemorrhagic shock, more than half of which were victims of penetrating trauma, was randomized to one of two fluid resuscitation protocols: target SBP $>100 \mathrm{~mm} \mathrm{Hg}$ or target SBP of $70 \mathrm{~mm} \mathrm{Hg}$. And fluid therapy was titrated to this endpoint until definitive hemostasis was achieved. His results showed similar survival rates in both groups suggesting that resorting to hypotensive resuscitation did not affect mortality [22]. Nevertheless, since then, more evidence has emerged favoring the concept of hypotensive resuscitation. Morrison et al. performed a randomized controlled study on 90 patients that required a laparotomy or thoracotomy following trauma; they were divided into 2 groups; the first group was resuscitated aiming for a mean arterial blood pressure of $65 \mathrm{~mm} \mathrm{Hg}$ with standard fluid protocol, while the second group was managed by hypotensive resuscitation to a mean blood pressure of $50 \mathrm{~mm} \mathrm{Hg}$. The results showed that the second group had a lower requirement of fluid and blood product transfusion, lower postoperative mortality, and reduced postoperative bleeding. And in those of them who did develop a coagulopathy, it was less severe compared to coagulopathic patients of the first group as evidenced by INR measurements [23].

The ratio of packed $\mathrm{RBC}$ (PRBC) : fresh frozen plasma $(\mathrm{FFP})$ : platelets (plt) should be $1: 1: 1$. Two studies showed that there was an increase in survival rate by increasing the ratio of FFP to RBC [24] and platelets to RBC [25]. This is the current military guidelines in massive transfusion [9].

3.5. Antifibrinolytics. Crash 2 was a randomized controlled trial, that was performed in 274 hospitals in 40 different countries and involved 20,211 patients to assess the effect of tranexamic acid on death, vasoocclusive events, and blood transfusion in trauma patients. The patients had to be above the age of 16 years, exposed to trauma, and had to have evidence of bleeding in the form of systolic blood pressure of less than $90 \mathrm{~mm} \mathrm{Hg}$ or a pulse rate of 110 beats per minute. 10,060 patients received tranexamic acid and 10,067 were randomized to the placebo group. The results yielded that tranexamic acid reduced the mortality rate to $14.5 \%$ compared to $16 \%$ in the placebo group. The conclusion of this trial was that tranexamic acid reduced the risk of death in trauma patients that were at risk of hemorrhage [26].

This conclusion was also echoed by Morrison et al. in the Military Application of Tranexamic Acid in Trauma Emergency Resuscitation study (MATTER) in 2012. The study was designed as a retrospective observational study that looked at a cohort of 896 patients admitted with combat injuries; the cohort was primarily divided into two groups the first included 293 patients who received TXA, and the second included the rest of the cohort who did not receive TXA. Both groups were further subdivided based on whether patients received massive transfusion (identified as 10 or more units of PRBCs within 24 hours). The outcomes that were measured were mortality at 24 hours, 48 hours, and 30 days, as well as the influence of TXA administration on postoperative coagulopathy and the rate of thromboembolic complications. The results showed an absolute reduction of in-hospital mortality by $6.5 \%$ in the TXA group compared to the non-TXA group and an absolute reduction of $49 \%$ in the TXA massive transfusion group compared to the massive transfusion non-TXA group. This was not completely cost-free though, as the study established that there was a higher rate of thromboembolic events in the TXA and TXA massive transfusion groups compared to their nonTXA counterparts, but it was noteworthy that there were no increased fatalities due to those events.

\section{Hemorrhage Control and Damage Control Surgery}

Despite taking all the above measures, if hemorrhage control is not achieved these resuscitation efforts would be futile. Hemorrhage control should be achieved in a timely fashion, initially by temporary measures such as direct compression, hemostatic dressings, tourniquets or the application of pelvic binders, and finally by definitive surgical control of the source of bleeding.

Temporary measures like the tourniquet, is well recognized and was first documented in 1674. Bellamy believed that $38 \%$ of the patients in the Vietnam War that died from extremity hemorrhage could have been salvaged by appropriate application of a tourniquet [27]. Pelvic binders help stabilize exsanguinating patient with open pelvic fractures by reducing their pelvic volume thus increasing intrapelvic pressure providing a tamponade effect to the catastrophic bleeding that is associated with these injuries. Extreme caution needs to be taken with both of these methods as they are both associated with grave complications due to prolonged application; tourniquets can cause neuropraxia, limb ischemia muscle injury, and compartment syndrome. On the other hand, pelvic binders can lead to skin necrosis.

Damage control surgery is a concept that emerged because the physiological derangements of hemorrhagic shock are more important than definitive surgery. It was described by Feliciano et al. as a 3-stage procedure in which the first is to control the bleeding, the second stage is where the patient is transferred to ITU for correction of the lethal triad and stabilization, and the 3rd stage is for definitive surgical repair [28].

\section{Conclusion}

Whilst massive hemorrhage continues to be a major cause of mortality, it is often reversible, and it can be adequately managed by early identification of these patients and the source of their bleeding, approaching them with (CABC) protocol and prevention and treatment of the lethal triad and damage control surgery to stop the bleeding.

\section{Conflict of Interests}

The authors declare that there is no conflict of interests regarding the publication of this paper. 


\section{References}

[1] D. S. Kauvar, R. Lefering, and C. E. Wade, "Impact of hemorrhage on trauma outcome: an overview of epidemiology, clinical presentations, and therapeutic considerations," Journal of Trauma, vol. 60, no. 6, pp. S3-S11, 2006.

[2] R. L. Gruen, G. J. Jurkovich, L. K. McIntyre, H. M. Foy, and R. V. Maier, "Patterns of errors contributing to trauma mortality: lessons learned from 2594 deaths," Annals of Surgery, vol. 244, no. 3, pp. 371-378, 2006.

[3] The American College of Surgeons, "Shock," in Advanced Trauma Life Support, The American College of Surgeons, Ed., pp. 59-73, 1990.

[4] R. H. Birkhahn, T. J. Gaeta, D. Terry, J. J. Bove, and J. Tloczkowski, "Shock index in diagnosing early acute hypovolemia," American Journal of Emergency Medicine, vol. 23, no. 3, pp. 323-326, 2005.

[5] C. M. Cannon, C. C. Braxton, M. Kling-Smith, J. D. Mahnken, E. Carlton, and M. Moncure, "Utility of the shock index in predicting mortality in traumatically injured patients," Journal of Trauma, vol. 67, no. 6, pp. 1426-1430, 2009.

[6] M. W. Ardagh, T. Hodgson, L. Shaw, and D. Turner, "Pulse rate over pressure evaluation (ROPE) is useful in the assessment of compensated haemorrhagic shock," Emergency Medicine, vol. 13, no. 1, pp. 43-46, 2001.

[7] R. Campbell, M. W. Ardagh, and M. Than, "validation of pulse rate over pressure evaluation index as a detector of early occult hemorrhage," Journal of Trauma and Acute Care Surgery, vol. 73, no. 1, pp. 286-288, 2012.

[8] C. W. Kam, C. H. Lai, S. K. Lam, F. L. So, C. L. Lau, and K. H. Cheung, "What are the ten new commandments in severe polytrauma management?" World Journal of Emergency Medicine, vol. 1, no. 2, 2010.

[9] R. Dawes and G. R. Thomas, "Battlefield resuscitation," Current Opinion in Critical Care, vol. 15, no. 6, pp. 527-535, 2009.

[10] A. Ferrara, J. D. MacArthur, H. K. Wright, I. M. Modlin, and M. A. McMillen, "Hypothermia and acidosis worsen coagulopathy in the patient requiring massive transfusion," American Journal of Surgery, vol. 160, no. 5, pp. 515-518, 1990.

[11] L. M. Gentilello and D. J. Pierson, "Trauma critical care," American Journal of Respiratory and Critical Care Medicine, vol. 163, no. 3, pp. 604-607, 2001.

[12] Z. Arthurs, D. Cuadrado, A. Beekley et al., "The impact of hypothermia on trauma care at the 31st combat support hospital," American Journal of Surgery, vol. 191, no. 5, pp. 610614, 2006.

[13] Z. H. Meng, A. S. Wolberg, D. M. Monroe III, and M. Hoffman, "The effect of temperature and $\mathrm{pH}$ on the activity of factor VIIa: implications for the efficacy of high-dose factor VIIa in hypothermic and acidotic patients," Journal of Trauma, vol. 55, no. 5, pp. 886-891, 2003.

[14] W. Z. Martini, M. A. Dubick, A. E. Pusateri, M. S. Park, K. L. Ryan, and J. B. Holcomb, "Does bicarbonate correct coagulation function impaired by acidosis in swine?" Journal of Trauma, vol. 61, no. 1, pp. 99-106, 2006.

[15] J. W. Davis, K. L. Kaups, and S. N. Parks, "Base deficit is superior to $\mathrm{pH}$ in evaluating clearance of acidosis after traumatic shock," Journal of Trauma, vol. 44, no. 1, pp. 114-118, 1998.

[16] H. Lier, H. Krep, S. Schroeder, and F. Stuber, "Preconditions of hemostasis in trauma: a review. The influence of acidosis, hypocalcemia, anemia, and hypothermia on functional hemostasis in trauma," Journal of Trauma, vol. 65, no. 4, pp. 951960, 2008.

[17] J. R. Hess, K. Brohi, R. P. Dutton et al., "The coagulopathy of trauma: a review of mechanisms," Journal of Trauma, vol. 65, no. 4, pp. 748-754, 2008.

[18] K. Brohi, J. Singh, M. Heron, and T. Coats, "Acute traumatic coagulopathy," Journal of Trauma, vol. 54, no. 6, pp. 1127-1130, 2003.

[19] R. Davenport and S. Khan, "Management of major trauma haemorrhage: treatment priorities and controversies," British Journal of Haematology, vol. 155, no. 5, pp. 537-548, 2011.

[20] M. D. Duke, C. Guidry, J. Guice et al., "Restrictive fluid resuscitation in combination with damage control resuscitation: time for adaptation," Journal of Trauma and Acute Care Surgery, vol. 73, no. 3, pp. 674-678, 2012.

[21] D. J. Dries, "Hypotensive resuscitation," Shock, vol. 6, no. 5, pp. 311-316, 1996.

[22] R. P. Dutton, C. F. Mackenzie, and T. M. Scalea, "Hypotensive resuscitation during active hemorrhage: impact on in-hospital mortality," Journal of Trauma, vol. 52, no. 6, pp. 1141-1146, 2002.

[23] C. A. Morrison, M. M. Carrick, M. A. Norman et al., "Hypotensive resuscitation strategy reduces transfusion requirements and severe postoperative coagulopathy in trauma patients with hemorrhagic shock: preliminary results of a randomized controlled trial," Journal of Trauma, vol. 70, no. 3, pp. 652-663, 2011.

[24] M. A. Borgman, P. C. Spinella, J. G. Perkins et al., "The ratio of blood products transfused affects mortality in patients receiving massive transfusions at a combat support hospital," Journal of Trauma, vol. 63, no. 4, pp. 805-813, 2007.

[25] J. G. Perkins, A. P. Cap, and P. C. Spinella, "An evaluation of the impact of apheresis platelets used in the setting of massively transfused trauma patients," Journal of Trauma, vol. 66, no. 4, pp. S77-S85, 2009.

[26] CRASH II, "Clinical randomisation of an antifibrinolytic in significant haemor- rhage," http://www.crash2.lshtm.ac.uk.

[27] R. F. Bellamy, "The causes of death in conventional land warfare: implications for combat casualty care research," Military Medicine, vol. 149, no. 2, pp. 55-62, 1984.

[28] D. V. Feliciano, J. M. Burch, V. Spjut-Patrinely, K. L. Mattox, and G. L. Jordan Jr., "Abdominal gunshot wounds. An urban trauma center's experience with 300 consecutive patients," Annals of Surgery, vol. 208, no. 3, pp. 362-370, 1988. 


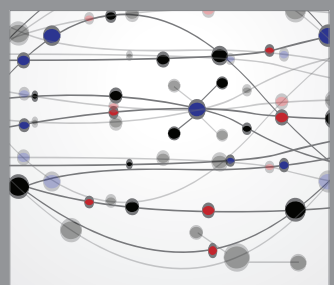

The Scientific World Journal
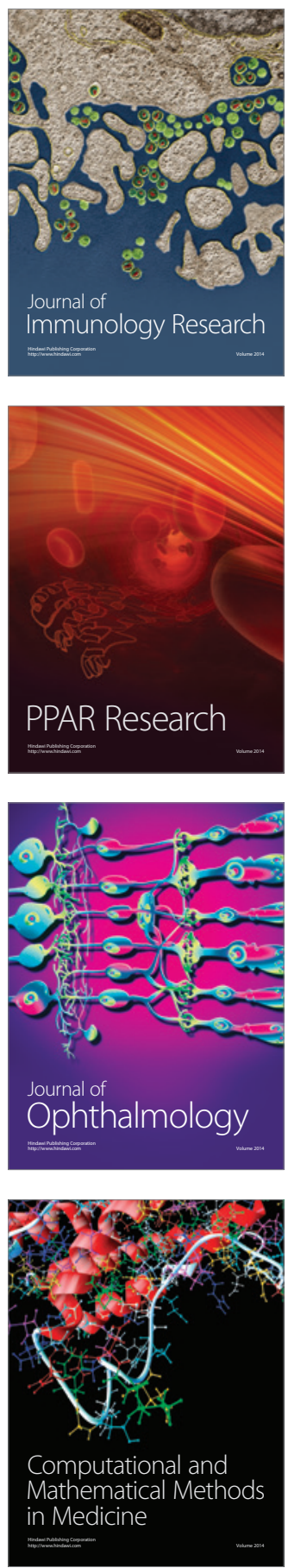

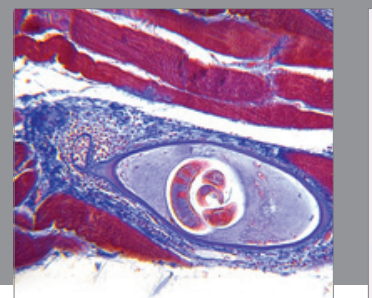

Gastroenterology

Research and Practice
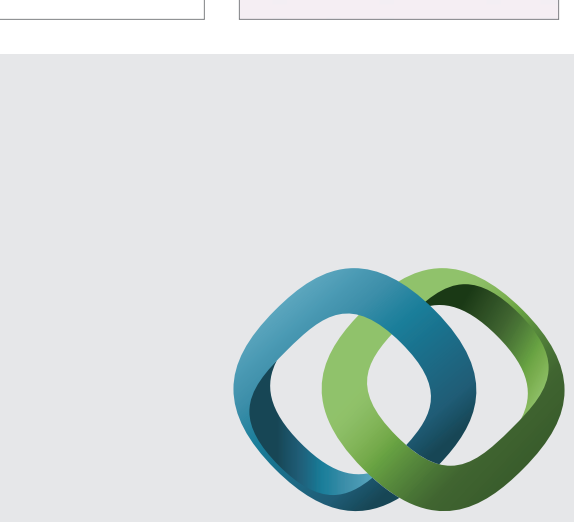

\section{Hindawi}

Submit your manuscripts at

http://www.hindawi.com
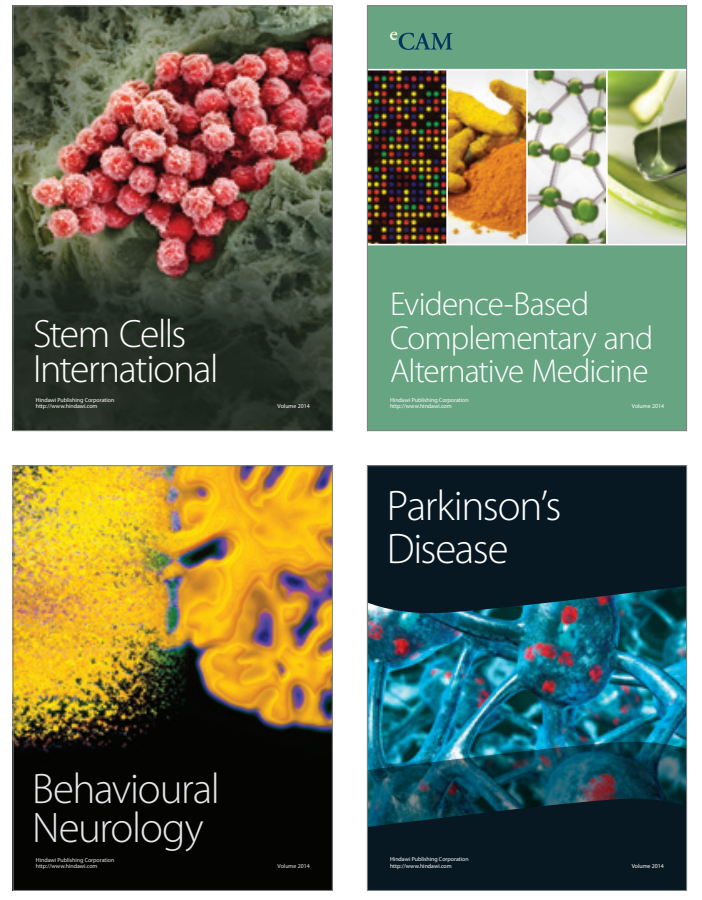
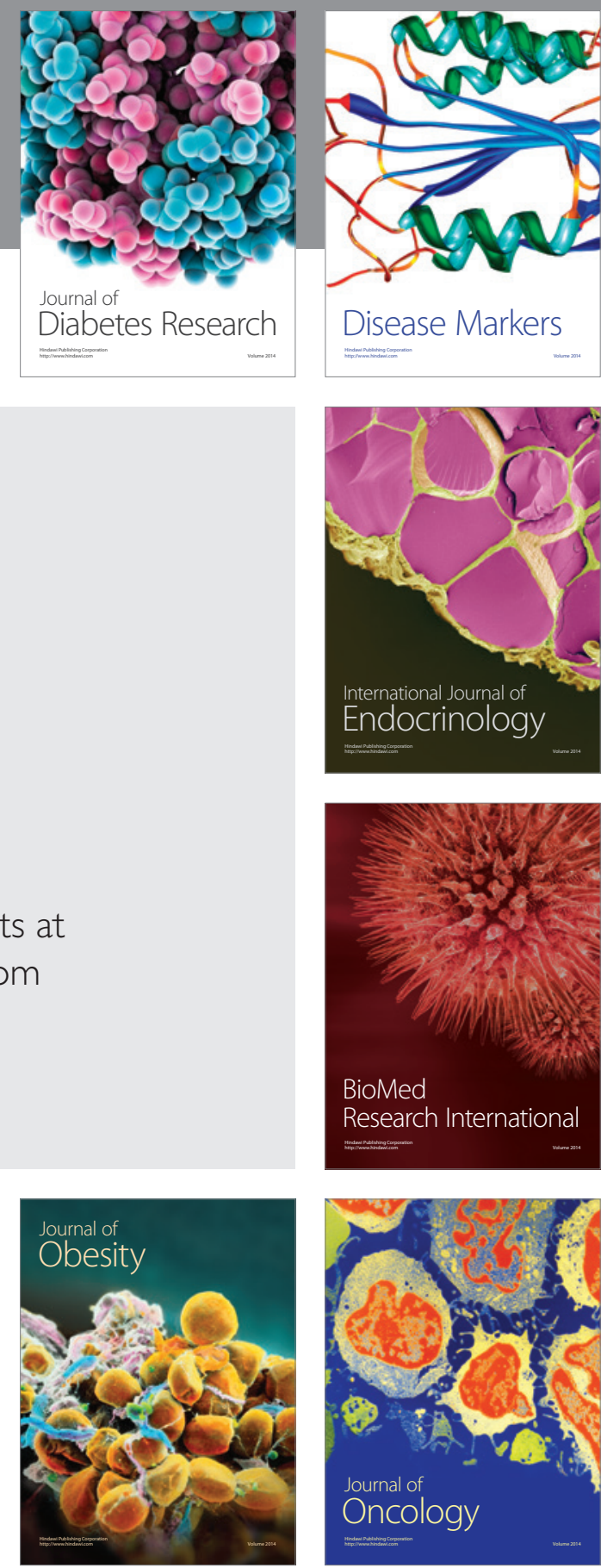

Disease Markers
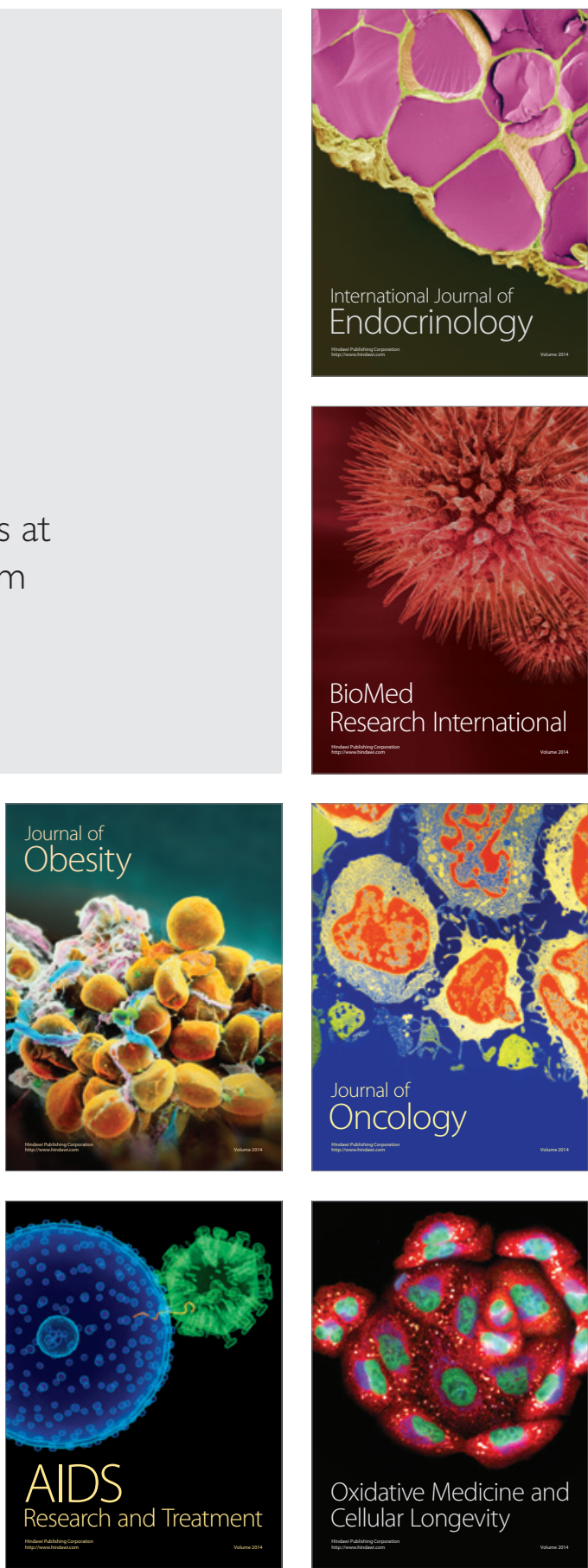